\title{
JOGOS CONCEITUAIS EM AMBIÊNTE ESPECÍFICO PARA ENSINO E APERFEIÇOAMENTO DE UMA EQUIPE SUB 14 FEMININA DE VOLEIBOL
}

\author{
JOGOS CONCEITUAIS NO VOLEIBOL
}

\author{
${ }^{1,}{ }^{2}$ Marcelo Francisco Rodrigues, ${ }^{2,3,4}$ Henrique Miguel, ${ }^{3}$ Marcus Vinicius de Almeida Campos.
}

\section{RESUMO}

Introdução: O Voleibol tem como uma das características mais importantes, a eficácia dos fundamentos para que as equipes desempenhem com sucesso, sua participação nos jogos. Diante das especificidades do Voleibol, como por exemplo: onde não há a interação com o adversário, não ocorre à retenção da bola durante as ações do jogo e os objetivos são comuns entre às equipes, de colocar a bola no solo adversário. Objetivo: O objetivo do referido estudo, teve como objetivo, analisar o desenvolvimento da equipe de Voleibol Feminina sub 14, da cidade de Aguaí/SP submetida a intervenções dos jogos conceituais em ambiente específico durante um campeonato regional. Método: Foi realizada uma análise descritiva contínua dos resultados dos jogos da equipe. Participaram deste estudo, 20 atletas do sexo feminino com idade média de 13,6 \pm 0,64 anos da categoria mirim, que disputa campeonatos regionais e estaduais, com vivência média de 3 anos no voleibol. As intervenções foram realizadas durante as aulas, onde foram propostos jogos conceituais de ambiente específico, com o objetivo de aperfeiçoar a aprendizagem dos fundamentos técnicos e ações táticas do voleibol, onde, trabalharam situações $3 \times 3,4 \times 4,5 \times 5$ e $6 \times 6$, com a manipulação das dimensões da quadra, das regras do jogo, e dinâmica dos mesmos. As intervenções ocorreram durante cinco semanas com duas aulas semanais de voleibol com duração de duas horas. Resultados e Discussão: A análise descritiva quantitativa e discreta como também foram realizadas, análises sobre o desempenho da equipe durante os jogos, inicialmente após a primeira derrota no jogo 1, identificamos um número grande erros nos fundamentos saque e recepção, houve também um grande desequilíbrio nos fundamentos ataque e recepção. Diante deste quadro, foram elaborados jogos conceituais em ambiente específico para a melhora destes fundamentos acima citados. Já na análise utilizando teste $t$ de student para comparação de sets vencidos e perdidos, encontramos diferenças estatísticas nos fundamentos saque e recepção. Conclusões: Concluímos que os jogos conceituais em ambiente específicos propostos foram preponderantes para a melhora da performance da equipe, tendo a recepção e o saque como fundamentos que determinarão o sucesso e insucesso da equipe durante os jogos.

Palavras Chaves: voleibol; jogos reduzidos; treinamento.

\section{ABSTRACT}

Introduction: Volleyball has one of the most important characteristics, the effectiveness of the fundamentals for teams to successfully play their part in games. Given the specifics of Volleyball, such as where there is no interaction with the opponent, does not occur to the retention of the ball during the actions of the game and goals are common among the teams, to put the ball in the opponent's ground. Objective: The aim of this study was to analyze the development of the sub - 14 women 's volleyball team in the city of Aguaí, SP, Brazil, submitted to conceptual game interventions in a specific environment during a regional championship. Method: A continuous descriptive analysis of team game results was performed. Twenty female athletes with a mean age of $13.6 \pm 0.64$ years in the junior category, who compete in regional and state championships, with an average age of 3 years in volleyball participated in this study. The interventions were carried out during classes, where specific environmental games were proposed, with the objective of improving the learning of the technical fundamentals and tactical actions of volleyball, where $3 \times 3,4 \times 4,5 \times 5$ and $6 \times 6$ situations were handled, with the manipulation of the dimensions of the court, the rules of the game, and their dynamics. Interventions occurred for five weeks with two weekly volleyball classes lasting two hours. Results and Discussion: The quantitative and discrete descriptive analysis were also performed, analyzes on the performance of the team during the games, initially after the first defeat in game 1 , we identified a large number of mistakes in the grounds serve and reception, there was also a large imbalance in the basics attack and reception. In this context, conceptual games were developed in a specific environment to improve these fundamentals. Already in the analysis using Student's test for comparison of overdue and lost sets, we found statistical differences in the grounds of serve and reception. Conclusions: We conclude that the proposed conceptual games in specific environments 
were predominant in order to improve the performance of the team, with reception and serve as fundamentals that will determine the success and failure of the team during the games

Keywords: volleyball; reduced games; training.

1-Departamento de Educação Física - UNIMOGI

2-Departamento de Educação Física Unipinhal , 3 - Departamento de Educação Física - FEUC, 4 - Departamento de educação física UNIFENAS.

Endereço para correspondência do $1^{\circ}$ autor: Marcelo Francisco Rodrigues

UNIMOGI: Avenida Padre Jaime, 2.600. Jardim Serra Dourada, Mogi Guaçu/SP. Tel (19) 3831- 3080.

E-mail: marcelo.edufisica@hotmail.com, prhmiguel@gmail.com, mvacampos@gmail.com .

\section{INTRODUÇÃO}

O Voleibol tem como uma das características mais importantes, a eficácia dos fundamentos para que as equipes desempenhem com sucesso sua participação nos jogos (MESQUITA, et al., 2001; LIMA et al., 2010) Diante das especificidades do Voleibol, como por exemplo: onde não há a interação com o adversário, não ocorre a retenção da bola durante as ações do jogo e os objetivos são comuns entre ambas as equipes, de colocar a bola no solo da equipe adversária (MESQUITA, 1996). Diante disso, a perfeição das execuções das ações técnicas fundamentais do voleibol caracteriza a maior parte do processo de ensino aprendizagem do jogo, bem como a planificação do treinamento. (COLLET et al., 2007).

Atualmente, os treinadores têm procurado cada vez mais ferramentas para que suas equipes sejam mais eficazes durante o jogo. No Voleibol, a ciência tem colaborado para elaboração de novas ferramentas de análises do jogo como um todo, buscando entender o jogo e quais os detalhes que diferenciam ganhadores de perdedores (GRECCO e MATHIAS, 2009).

Dentre as opções de avaliação e quantificação do jogo de voleibol o scout manual é um dos mais utilizados pelos treinadores de voleibol principalmente para equipes de categorias de base, devido à questão financeira e aplicabilidade não só durante e após a partida, bem como elaboração das sessões de treinamento diante dos resultados dos jogos (HAIACHI et al., 2014; MARQUES JUNIOR e ARRUDA, 2016)

Dominar com eficiência o como fazer do jogo, representado pelas ações técnicas, juntamente com a tomada de decisão correta em cada momento específico do jogo faz com que a partida ocorra de maneira satisfatória para quem domina esses conceitos (GARGANTA e OLIVEIRA, 1996). Deste modo Graça e Mesquita (2002), propõem que o ensino, desde o início do voleibol, deve preconizar 0 entendimento das questões técnico táticas com ações estruturadas de forma progressiva e sequencial, próximas as ações do jogo, sendo os jogos reduzidos um viés de ferramenta de trabalho nesse sentido.

Ainda segundo Graça e Mesquita (2002), em sua investigação sobre o ensino do jogo, não há um consenso sobre a forma mais eficaz de ensinar o voleibol, por conta da exigência de eficiência na execução dos movimentos técnicos do esporte, fazendo com que a repetição sistemática dos fundamentos, ainda continue sendo o modo mais seguro de aprendizagem do jogo de voleibol por parte de alguns treinadores.

Diante dessa premissa a que emerge a prática do Voleibol como um jogo coletivo, Scaglia (2011), nos diz que o jogo é um sistema caótico, onde possui um padrão estruturado de acontecimentos pautados na lógica interna do mesmo, onde os jogos conceituais em ambientes específicos podem ser uma saída interessante para que o aluno que aprende, aprende conjuntamente com as emergências que surgem durante a prática do jogo formal. Nesse contexto, buscamos investigar as possíveis influencias que os treinamentos a partir dos jogos conceituais teriam na eficácia da equipe de Voleibol sub 14 da cidade de Aguaí durante o campeonato regional.

\section{OBJETIVO GERAL}

A presente proposta de estudo, teve como objetivo, analisar o desenvolvimento da equipe de Voleibol Feminina sub 14, da cidade de Aguaí/SP submetida a intervenções dos jogos conceituais em ambiente específico durante um campeonato regional.

\section{OBJETIVOS ESPECÍFICOS}

$\checkmark$ Verificar a influência dos jogos conceituais nas variáveis analisadas.

$\checkmark \quad$ Identificar possíveis padrões dos erros cometidos durante os jogos com vitórias e derrotas.

$\checkmark$ Verificar correlações entre a eficácia das variáveis entre sets ganhos e perdidos. 


\section{JOGOS CONCEITUAIS NO VOLEIBOL}

\section{MÉTODO}

Esta pesquisa foi realizada utilizando - método misto composto por pesquisas qualitativas e quantitativas. Inicialmente com a pesquisa bibliográfica com 0 intuito de construir o referencial teórico do trabalho e para a discussão sobre os métodos e jogos a serem utilizados, bem como a discussão da pesquisa, confrontando os resultados encontrados com os já existentes na literatura específica. A segunda etapa da investigação foi realizada a partir de uma pesquisa de campo junto a equipe feminina infantil da cidade de Aguaí/SP com idade média de 13,6 $\pm 0,64$ anos, sendo uma equipe de representação, que participa de campeonatos regionais. Esta equipe foi selecionada diante da facilidade referente ao contato com o professor/pesquisador que também atuou como técnico desta equipe.

Os jogos conceituais em ambiente específico. As intervenções foram realizadas durante as aulas de treinamento de voleibol, onde foram propostas atividades, tendo como base metodológica os jogos conceituais. Portanto as atletas, passaram a praticar aulas pautadas nos jogos conceituais de ambiente específico, Scaglia (2011), com o objetivo de aperfeiçoar a aprendizagem dos fundamentos técnicos e ações táticas do voleibol, onde, trabalharam situações $3 \times 3,4 \times 4,5 \times 5$ e $6 \times 6$, com a manipulação das dimensões da quadra, das regras do jogo, e dinâmica dos mesmos. As intervenções ocorreram durante 5 semanas com duas aulas semanais de voleibol com duração de duas horas, onde os conceitos do jogo de vôlei

\section{Coleta de dados \\ Foi realizada uma análise} descritiva contínua dos resultados dos jogos da equipe ao longo do campeonato, relacionando com a apresentação dos conteúdos dos jogos conceituais aplicados durante as aulas (REIS e REIS., 2002). Referente ao método quantitativo, foi realizado através do método scout simples, que foram anotados a partir de imagens dos jogos, onde foram contabilizados os fundamentos realizados durante os jogos (Mathias e Grecco, 2009).

Participaram deste estudo, 20 atletas do sexo feminino com idade média de $13,6 \pm 0,64$ anos da categoria mirim, que disputa campeonatos regionais e estaduais, com vivência média de 3 anos no voleibol em um projeto municipal de esporte. Todas as atletas assinaram um termo de assentimento, e os responsáveis pelas atletas um terno de consentimento livre e esclarecido, referente a toda a pesquisa. Esta pesquisa é uma parte do projeto submetido e aprovado pelo comitê de ética do Instituto Piracicabano de Ensino da Igreja Metodista, número: 01207518.5.0000.5507

\section{Estatística}

Para verificação da normalidade do conjunto de dados de cada variável obtidos nos sets vencedores e perdedores a partir do scout realizado durante os jogos, foi utilizado o teste estatístico Lilliefours. Foi utilizado o teste $\mathrm{T}$ de student, para comparação dos resultados dos sets vencedores e perdedores nos jogos.

Delimitações da pesquisa

Os scouts apenas dos erros cometidos foram feitos por 2 alunos de Voleibol com média de conhecimento do voleibol de 4,5 anos, e o treinamento sobre os fundamentos a serem selecionados foram feitos em duas semanas antes do início do campeonato e foram separados da seguinte 6 rrma:

Saque: Quando o sacador golpeava a bola para fora da quadra adversária ou na rede e que a bola não passava para o lado adversário

Ataque: Quando o atacante atacava a bola diretamente para fora ou atacava na rede e a bola não passava para a quadra adversária.

Recepção: Toda a recepção que culminava em ponto do adversário, pós saque adversário ou quando o adversário devolvia a bola atacando ou apenas passando a bola.

Ressaltando que foram apenas utilizados os números de erros cometidos pela equipe, por conta da limitação de tempo de treinamento dos responsáveis pelos dados. Os resultados dos sets jogo a jogo foram anotados a partir desses scouts realizados.

\section{RESULTADOS E DISCUSSÃO}

Análise Descritiva Quantitativa

Abaixo, apresenta-se a tabela dos jogos que foram disputados pela equipe durante o campeonato, como também análise descritiva quantitativa e discreta (REIS e REIS, 2002). 
Tabela1: Resultados dos jogos analisados na pesquisa com os resultados sets por set durante os 7 jogos.

\begin{tabular}{l|llllll} 
& $\mathbf{1}^{\circ}$ SET & $\mathbf{2}^{\circ}$ SET & $\mathbf{3}^{\circ}$ SET & $4^{\circ}$ SET & $5^{\circ}$ SET & FINAL \\
\hline JOGO 1 & $25 \times 17$ & $08 \times 25$ & $20 \times 25$ & $25 \times 14$ & & $1 \times 3^{*}$ \\
JOGO 2 & $21 \times 25$ & $24 \times 26$ & $25 \times 16$ & $25 \times 13$ & $16 \times 14$ & $3 \times 2$ \\
JOGO 3 & $25 \times 9$ & $17 \times 25$ & $25 \times 19$ & $25 \times 19$ & & $3 \times 1$ \\
JOGO 4 & $25 \times 15$ & $25 \times 11$ & $25 \times 22$ & & $3 \times 0$ \\
JOGO 5 & $25 \times 13$ & $25 \times 15$ & $25 \times 8$ & & $3 \times 0$ \\
JOGO 6 & $25 \times 15$ & $24 \times 26$ & $25 \times 15$ & & & $3 \times 0$ \\
JOGO 7 & $25 \times 18$ & $15 \times 25$ & $17 \times 25$ & $13 \times 25$ & & $1 \times 3^{*}$
\end{tabular}

No decorrer do campeonato, foram feitas análises sobre o desempenho da equipe durante os jogos, inicialmente após a primeira derrota no jogo 1 , identificamos um número grande erros nos fundamentos saque e recepção, houve também um grande desequilíbrio nos fundamentos ataque e recepção. Diante deste quadro, foram elaborados jogos conceituais em ambiente específico para a melhora destes fundamentos acima citados. Os jogos tiveram como conceito as rebatidas através de manchetes com apenas dois toques por equipe, sendo elas divididas em meia quadra com 3 jogadores, pois este era a linha de passadores adotadas pela equipe. Outra intervenção feita a partir da observação dos erros do primeiro jogo foi a variabilidade de execução e diminuição dos erros de saque durante os treinos e jogos.

No segundo jogo, a questão da instabilidade nos erros de saques e passes seguiram-se, porém, a partir do $3^{\circ}$ set a equipe conseguiu diminuir os erros de saque, com isso dificultando a construção do ataque adversário e quando a bola era retornada, o aproveitamento do side - out, era consciente, sendo ou não a partir de ataques fortes ou apenas largadas. A partir daí os jogos foram formatados para que as situações de contraataque fossem privilegiadas, como por exemplo, no jogo 6x6, após a finalização de um ponto, mais uma bola era jogada para a equipe que tinha concluído o ponto para que houvesse mais uma possibilidade de contraataque e eram discutidas as decisões após o término dessas ações.

A seguir apresenta-se a tabela das médias dos erros cometidos dos jogos analisados da equipe durante o campeonato nos fundamentos Ataque, Recepção e Saque. As hipóteses que confirmam a hipótese nula foram representadas pelo símbolo *.

Tabela 2: Médias dos erros das variáveis nos sets vencidos e perdido durante os jogos analisados

\begin{tabular}{l|r}
\multicolumn{1}{c}{ SETS VENCIDOS } \\
\hline ATAQUE & $\mathbf{2 , 7 0} \pm \mathbf{1 , 2 1}$ \\
RECEPÇÃO & $\mathbf{4 , 5 3} \pm \mathbf{2 , 4 2}$ \\
SAQUE & $\mathbf{2 , 1 2} \pm \mathbf{1 , 3 2}$
\end{tabular}

SETS PERDIDOS

$2,64 \pm 1,86$
$6,89 \pm 3.03$
$3,27 \pm 1,42$

Marcelino et al., (2008), em sua analise do campeonato mundial de voleibol masculino de 2005, observou que é de extrema importância para continuidade positiva das equipes de alto nível, tanto do primeiro ao ultimo set, a continuidade do jogo a partir de uma boa recepção, o que pudemos observar nitidamente para esta equipe, a diminuição dos erros desse fundamento mostrou-se de extrema importância para vencer os sets.

No estudo citado acima, o autor encontrou diferenças estatísticas para o fundamento saque, como um fator preponderante para o sucesso das equipes vencedoras daquele mundial, corroborando também com os números encontrados nesta 
pesquisa, quanto menor o número erros de saque da equipe, maiores foram às chances de êxito no jogo. Marques Junior (2013), em seu trabalho, analisando eficácia dos fundamento do voleibol de homens e mulheres juvenis, que o erro de saque é um fator preponderante para que as equipes fossem derrotadas no set, variável correspondente para esta equipe apresentada neste estudo que também encontrou muita dificuldade na

\section{CONCLUSÃO}

Diante da especificidade do Voleibol de competição, novas possibilidades de ensino e aprendizagem desse esporte são necessárias, ainda neste sentido, a alta demanda cognitiva que o jogo exige do atleta, sendo que a tomada de decisão é de extrema importância para o sucesso no jogo, portanto, partindo desta premissa, os jogos reduzidos, se mostraram para esta equipe analisada um viés positivo de entendimento do jogo. Observou-se que a vivência dos jogos conceituais em ambiente específico contribuíra para a melhora significativa da equipe investigada, sendo creditada esta melhora, por conta das inúmeras vivências nas situações propostas, com isso as atletas, já administravam melhor as ações técnicas do jogo durante as partidas, como por exemplo, na diminuição dos erros de saque e recepção. Os jogos conceituais em ambiente específico, primeira apresentação da mesma equipe corroborando com a diferença estatística que confirma a hipótese nula na tabela supracitada. Neste mesmo trabalho também foram evidenciados que os saques que causavam dificuldade de deslocamento da linha de recepção, o que pudemos observar também para a equipe analisada com a diminuição dos seus erros de saque da equipe.

abordados nas intervenções trataram principalmente de situações diversas que ocorrem durante o jogo propriamente dito, que justificam a diminuição dos erros da recepção e saques errados, durante o jogo. Para o saque, durante as intervenções, foram incentivados os saques em posições estratégicas da quadra, juntamente com a liberdade de escolha na intensidade do mesmo, onde as atletas entenderam que este fundamento já atuava como uma ação ofensiva em prol da equipe. Contudo, mais pesquisas são necessárias utilizando os jogos conceituais em ambiente específico, como ferramenta de aprendizagem e treinamento no Voleibol que com certeza serão benéficos, levando em consideração as matrizes dos jogos como parte relevante para a planificação tanto na aprendizagem quanto no aperfeiçoamento do esporte.

\section{REFERÊNCIAS}

COLLET, C., NASCIMENTO, J. V., RAMOS, V., STEFANELLO, J. M. F. Construção e validação do instrumento de avaliação do desempenho técnico-tático no voleibol. Revista brasileira de Cineantropometria e Desempenho Humano, v. 13, n. 1, pag. 43, 2007.

GARGANTA, J.; OLIVEIRA, J. Estratégia e táctica nos jogos desportivos coletivos. In: OLIVEIRA, J.; TAVARES, F. (Eds.). Estratégia e táctica nos jogos desportivos coletivos. Porto: CED. 1996.

GRAÇA, A.S, MESQUITA I.R. A investigação sobre o ensino dos jogos desportivos: ensinar e aprender as habilidades básicas do jogo. Revista Portuguesa de Ciências do Desporto, v. 2, pag $67-79,2002$.

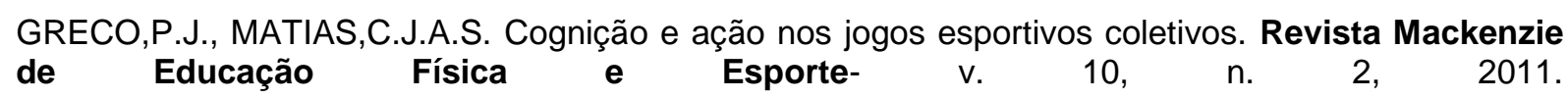

LIMA, C. O. V., MATIAS, C. J. A. S., GRECO, P. J. O conhecimento tático produto de métodos de ensino combinados e aplicados em sequências inversas no voleibol. Revista brasileira de Educação Física e Esporte, São Paulo, v. 26, n. 1, p. 47-129, Jan./Mar. 2012.

MARCELINO, R.; MESQUITA, I.; AFONSO, J. The weight of terminal actions in volleyball: contributions of the spike, serve and block for the teams' rankings in the World League'2005. International Journal of Performance Analysis in Sport, Cardiff, v. 8, n. 2, p. 1- 7, 2008

MARCELINO, R; MESQUITA, I; SAMPAIO, J; Estudo dos indicadores de rendimento em voleibol. Revista Brasileira de Educação Física e Esporte, São Paulo, v.24, n.1, p. 69-78, jan./mar. 201 
MARQUES JUNIOR, N.; EVIDÊNCIAS CIENTÍFICAS SOBRE OS FUNDAMENTOS DO VOLEIBOL: Importância desse conteúdo para prescrever o treino. Revista Brasileira de Prescrição e Fisiologia do Exercício. v. 7, n. 37, p.78-97. Jan/Fev. 2013

MARQUES JUNIOR, N.; ARRUDA, D. Análise do jogo de voleibol: ensino da execução dessa tarefa com Excel®. Revista Brasileira de Prescrição e Fisiologia do Exercício. v. 10. n. 57. p. 112-130, 2016

MATIAS, C.J.A.S.; GRECO, P.J. Análise de jogo nos esportes coletivos: o exemplo do voleibol. Revista Pensar a Prática, Goiânia, v.12, n. 3, p. 1- 15, 2009.

MESQUITA, I et al., A relação entre a eficiência e a eficácia no domínio das habilidades técnicas em Voleibol. Revista Portuguesa de Ciências do Desporto, v. 1, n. 3, pag. 33-39, 2001.

REIS, E. A., REIS I. A. (2002) Análise Descritiva de Dados. Relatório Técnico do Departamento de Estatística da UFMG.

SCAGLIA, A J, et al,. A organização do processo de ensino em função da lógica do jogo e das competências essenciais para a aprendizagem dos jogos coletivos de invasão. Revista Portuguesa de Ciências do Desporto, Porto, v. 11, supl. 4, p. 89, 2011. 